\title{
Texture and Planar Anisotropy of $r$-Value in Duplex Stainless Steel Sheet
}

\author{
Jun-ichi Hamada ${ }^{1, *}$ and Hirofumi Inoue ${ }^{2}$ \\ ${ }^{1}$ Steel Research Laboratories, Nippon Steel Corporation, Futtsu 293-8511, Japan \\ ${ }^{2}$ Department of Materials Science, Graduate School of Engineering, Osaka Prefecture University, Sakai 599-8531, Japan
}

\begin{abstract}
The effect of cold-rolling reduction on texture and planar anisotropy of the $r$-value was investigated in type 329J4L duplex stainless steel (DSS) sheet, which has a two-phase ferrite $(\alpha)$ and austenite $(\gamma)$ microstructure. In addition, the possibility of predicting the planar anisotropy of the $r$-value on the basis of the Taylor theory was investigated in consideration of the texture gradient through the thickness. An increase in coldrolling reduction resulted in the improved average $r$-value and the strong directionality with a maximum value at the diagonal direction. The $\alpha$ phase had an extremely strong $\alpha_{\mathrm{bcc}}$-fiber texture and the $\gamma$ phase had $\beta$ - and $\alpha_{\mathrm{fcc}}$-fiber textures. The texture gradient in the thickness direction was more remarkable in the $\alpha$ phase than in the $\gamma$ phase. The $r$-values calculated from the overall texture of the $\alpha$ phase using the relaxed constraints model with two relaxed shear strain constraints $e_{13}, e_{23}$ and a CRSS ratio of 1.1 for $\{112\}$ and $\{011\}\langle 111\rangle$ glide systems were in quite good agreement with the experimental $r$-values. This suggested that slip deformation in the $\alpha$ matrix was dominant for type 329J4L DSS sheet. However, the difference between the experimental and calculated results was remarkable in the vicinity of the diagonal direction. [doi:10.2320/matertrans.MG200908]
\end{abstract}

(Received September 28, 2009; Accepted November 18, 2009; Published March 25, 2010)

Keywords: duplex stainless steel, r-value, planar anisotropy, texture, orientation distribution function, Taylor model, cold rolling, annealing, microstructure

\section{Introduction}

Duplex stainless steels (DSS), such as type 329J4L, which have a two-phase ferrite $(\alpha)$ and austenite $(\gamma)$ microstructure, have been adopted for the fabrication of water tanks and heat exchange panels having complicated forms, because they have good corrosion resistance and strength. ${ }^{1-3)}$ In the case of using DSS in form of flat products, the formabilities of the cold-rolled and annealed sheets are important. Then, it is necessary to examine the microstructure and to understand its influence on the mechanical properties of the final product. It has been shown that the yield point and elongation of DSS cold-rolled and annealed sheets depend on the texture of the $\alpha$ matrix with the strong $\alpha$-fiber $(\{100\}\langle 011\rangle \sim\{111\}\langle 011\rangle)$ i.e., cold-rolling texture for BCC metal. ${ }^{4-13)}$ However, there are few studies on the planar anisotropy of the $r$-value and the effect of the texture of each phase on the $r$-value. ${ }^{7,8)}$

In this study, the texture and the planar anisotropy of the $r$-value were investigated for type 329J4L DSS sheets with different cold-rolling reductions. Additionally, we predicted the planar anisotropy of the $r$-value on the basis of the Taylor theory from the quantitative texture of each phase, and then the most suitable model for predicting the plastic anisotropy of DSS sheet was also examined by comparison with experimental data.

\section{Experimental Procedures}

The chemical composition of commercial type 329J4L hot-rolled plate used in this study is given in Table 1 . The thickness $(\mathrm{t})$ of the plate was $4.5 \mathrm{~mm}$. Hot-rolled specimens were annealed for $60 \mathrm{~s}$ at $1050^{\circ} \mathrm{C}$, followed by air cooling. The annealed specimen was cold-rolled to $67 \%, 78 \%$, and $89 \%$ reductions on a laboratory rolling mill, and the coldrolled sheets were annealed for $30 \mathrm{~s}$ at $1075^{\circ} \mathrm{C}$, followed by

*Formerly at Research \& Development Center, Nippon Steel \& Sumikin Stainless Steel Corporation
Table 1 Chemical composition of steel used (mass\%).

\begin{tabular}{ccccccccc}
\hline $\mathrm{C}$ & $\mathrm{Si}$ & $\mathrm{Mn}$ & $\mathrm{P}$ & $\mathrm{S}$ & $\mathrm{Ni}$ & $\mathrm{Cr}$ & $\mathrm{Mo}$ & $\mathrm{N}$ \\
\hline 0.02 & 0.5 & 0.7 & 0.02 & 0.003 & 6.9 & 25.2 & 3.0 & 0.11 \\
\hline
\end{tabular}

air cooling. The $r$-values of the JIS 13B test pieces cut from these sheets were measured after $10 \%$ tensile strain at angles of $0^{\circ}, 15^{\circ}, 30^{\circ}, 45^{\circ}, 60^{\circ}, 75^{\circ}$, and $90^{\circ}$ with respect to the rolling direction. The average $r$-value $\left(r_{\mathrm{m}}\right)$ was calculated using the following equation.

$$
r_{\mathrm{m}}=\left(r_{0}+2 r_{15}+2 r_{30}+2 r_{45}+2 r_{60}+2 r_{75}+r_{90}\right) / 12
$$

Also, in this study the anisotropic parameter $\left(A_{r}\right)$ was defined in the next expression,

$$
A_{r}=r_{\max }-r_{\min }
$$

where, $r_{\max }$ and $r_{\min }$ are the maximum and minimum values among the measured $r$-values in the seven directions, respectively. Microstructural observations were carried out by optical microscopy on the longitudinal section, or the section parallel to the rolling plane in the center of the final annealed specimens. The textures were quantitatively examined using the orientation distribution function (ODF). Incomplete pole figures of (200), (211), and (310) for the $\alpha$ phase and those of (200), (220), and (311) for the $\gamma$ phase were measured on the sections perpendicular to the normal direction (ND-section) and the transverse direction (TDsection) of the sheets by means of the Schultz reflection method using Mo K $\alpha$ radiation. From these pole figures, the ODF was determined using the iterative series-expansion method. ${ }^{14)}$ For estimation of the texture gradient in the sheets, the ND-sectional samples were prepared from various locations in the thickness direction. For measuring the average texture in the thickness direction of the sheets, a TD-sectional sample for pole figure measurement was prepared by stacking and bonding a large number of cut sheet samples. ${ }^{15,16)}$ The ODF on the TD-basis was trans- 


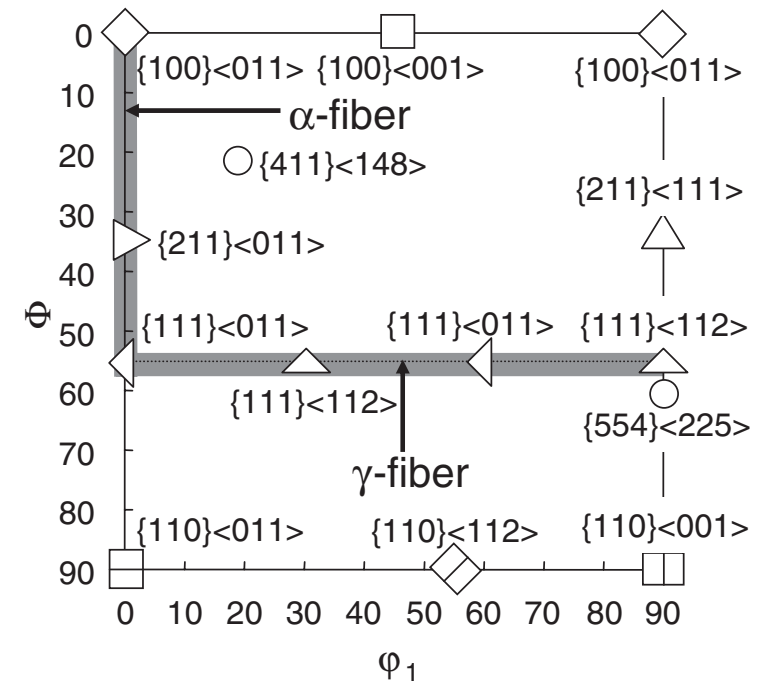

Fig. 1 Some important fiber textures and orientations in Euler space $\left(\varphi_{2}=45^{\circ}\right.$ section $)$.

formed into the normal ODF on the ND-basis by assuming orthorhombic sample symmetry. ${ }^{15)}$ Figure 1 shows the most important texture components and fibers for the $\alpha$ phase in the $\varphi_{2}=45^{\circ}$ section of ODF (Bunge's notation).

\section{Analytical Procedures}

In this study, the prediction of the $r$-value was carried out from ODF data using the Taylor full-constraints (FC) model ${ }^{17)}$ and the relaxed constraints (RC) model, ${ }^{18)}$ in which some shear-strain components are relaxed in contrast to those in a fully constrained state. Software developed by one of the authors was employed in this calculation. ${ }^{19-22)}$ In this study, the shear strain perpendicular to the tensile direction was taken as requisiteness for relaxing shear component, because it was thought that this component was hard to be most restricted in tensile testing, ${ }^{6,20,21)}$ and the necessity of the relaxation of the other components was examined. In other words, the RC23 model in which shear strain component $e_{23}$ is left free, RC1323 model in which $e_{13}$ and $e_{23}$ are left free, and RC121323 model in which $e_{12}, e_{13}$, and $e_{23}$ are left free $(1,2$, and 3 are defined as the tensile, width, and thickness directions, respectively) were used among several kinds of the RC models having the combination of shear strain components shown in Fig. 2. The RC1323 model, namely the pancake model, has been used as the most suitable model for BCC metals in the previous studies. ${ }^{6,16,20,23,24)}$ On the other hand, in FCC material, austenitic stainless steel and some aluminum alloy, it has been reported that the angular dependence of the yield strength or $r$-value are estimated by the FC model. ${ }^{6,19,22)}$ Assuming that the elongation in the tensile direction is $d \varepsilon$ and that the contractions in the width and thickness directions are $q d \varepsilon$ and $(1-q) d \varepsilon$, respectively, the deformation tensor can be defined as follows.

$$
E=\left[\begin{array}{ccc}
d \varepsilon & e_{12} & e_{13} \\
0 & -q d \varepsilon & e_{23} \\
0 & 0 & -(1-q) d \varepsilon
\end{array}\right]
$$

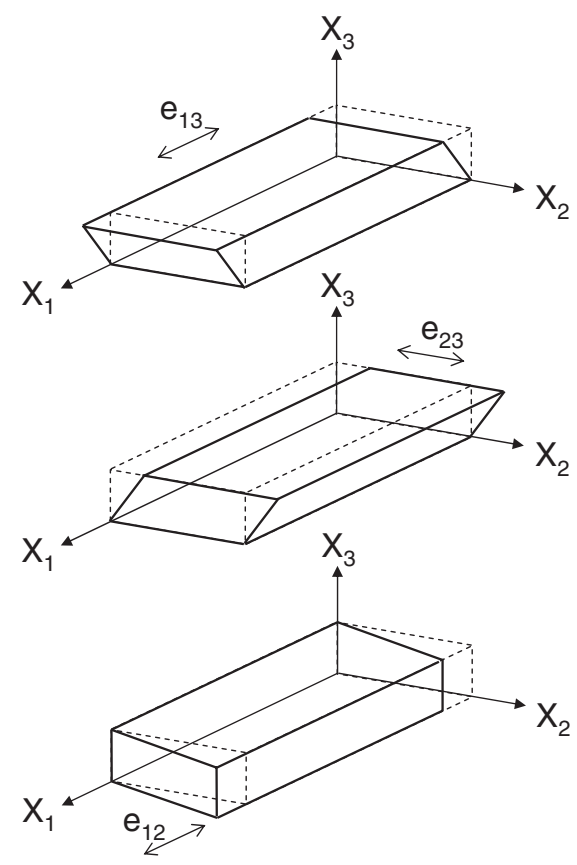

Fig. 2 Schematic representation of local shear strain relaxation in relaxedconstraints models. $X_{1}, X_{2}$ and $X_{3}$ are the tensile, width and thickness directions, respectively.

Consequently, the $r$-value is defined by the following equation.

$$
r=q /(1-q)
$$

In the FC model $e_{13}, e_{23}$ and $e_{12}$ are zero, in the RC23 model $e_{12}$ and $e_{13}$ are zero, and in the RC1323 model $e_{12}$ is zero. The $q$-value and $r$-value in each tensile direction can be calculated from the ODF by minimizing the plastic work of the slip deformation; the minimization can be performed by considering the combination of active slip systems. ${ }^{25,26)}$ The slip systems employed in this calculation were $\{110\}\langle 111\rangle$ and $\{211\}\langle 111\rangle$ for the $\alpha$ phase and $\{111\}\langle 011\rangle$ for the $\gamma$ phase. The values of the critical resolved shear stress (CRSS) ratio of the $\{211\}$ and $\{110\}$ glide planes $\left(\tau_{\{211\}} / \tau_{\{110\}}\right)$ are assumed to be $0.9,1.0$, and 1.1 in calculations for the $\alpha$ phase using the RC model.

\section{Results and Discussions}

\section{1 $R$-values}

Figure 3 shows the effect of cold-rolling reductions on the planar anisotropies of the $r$-values measured for the final annealed sheets. All specimens cold-rolled to different reductions showed a strong directionality, i.e. a noticeable planar anisotropy with the so-called reverse $\mathrm{V}$ type that had a maximum value at the $45^{\circ}$ direction. With an increase in the cold-rolling reduction, the $r$-value, in particular, increased in the vicinity of the $45^{\circ}$ direction. Figure 4 shows the effect of cold-rolling reduction on the average $r$-value. The average $r$ value also increased with increasing cold-rolling reduction. In this figure, the published experimental data of ferritic single-phase stainless steel sheets ${ }^{27,28)}$ were plotted, too. It is not very meaningful to make a quantitative comparison of the $r$-value between type 329J4L and ferritic single-phase 


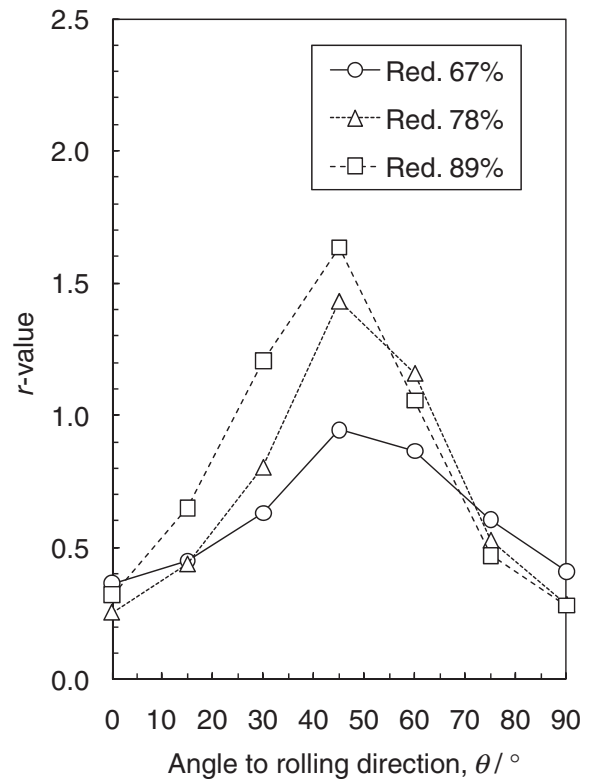

Fig. 3 Planar anisotropy of the $r$-value measured for final annealed sheets.

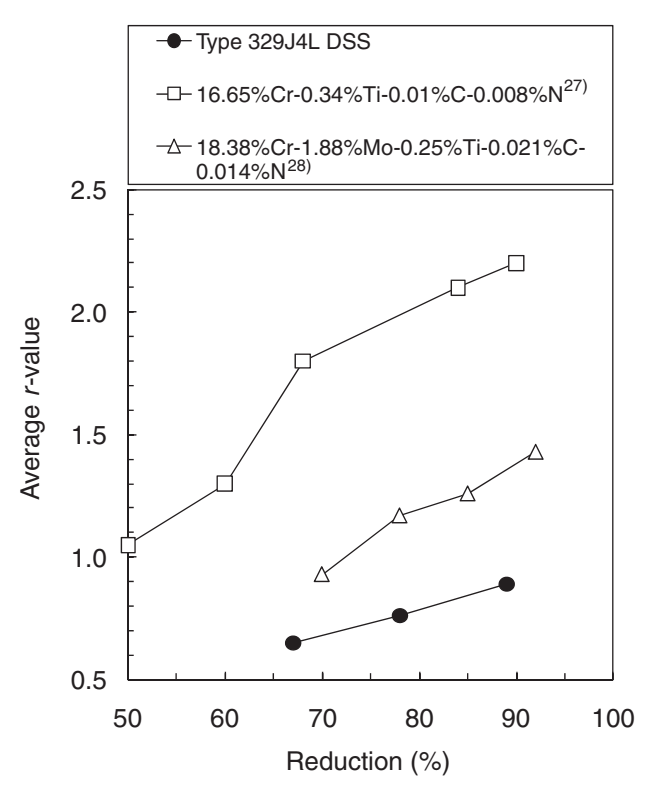

Fig. 4 Effect of cold-rolling reductions on average $r$-value.
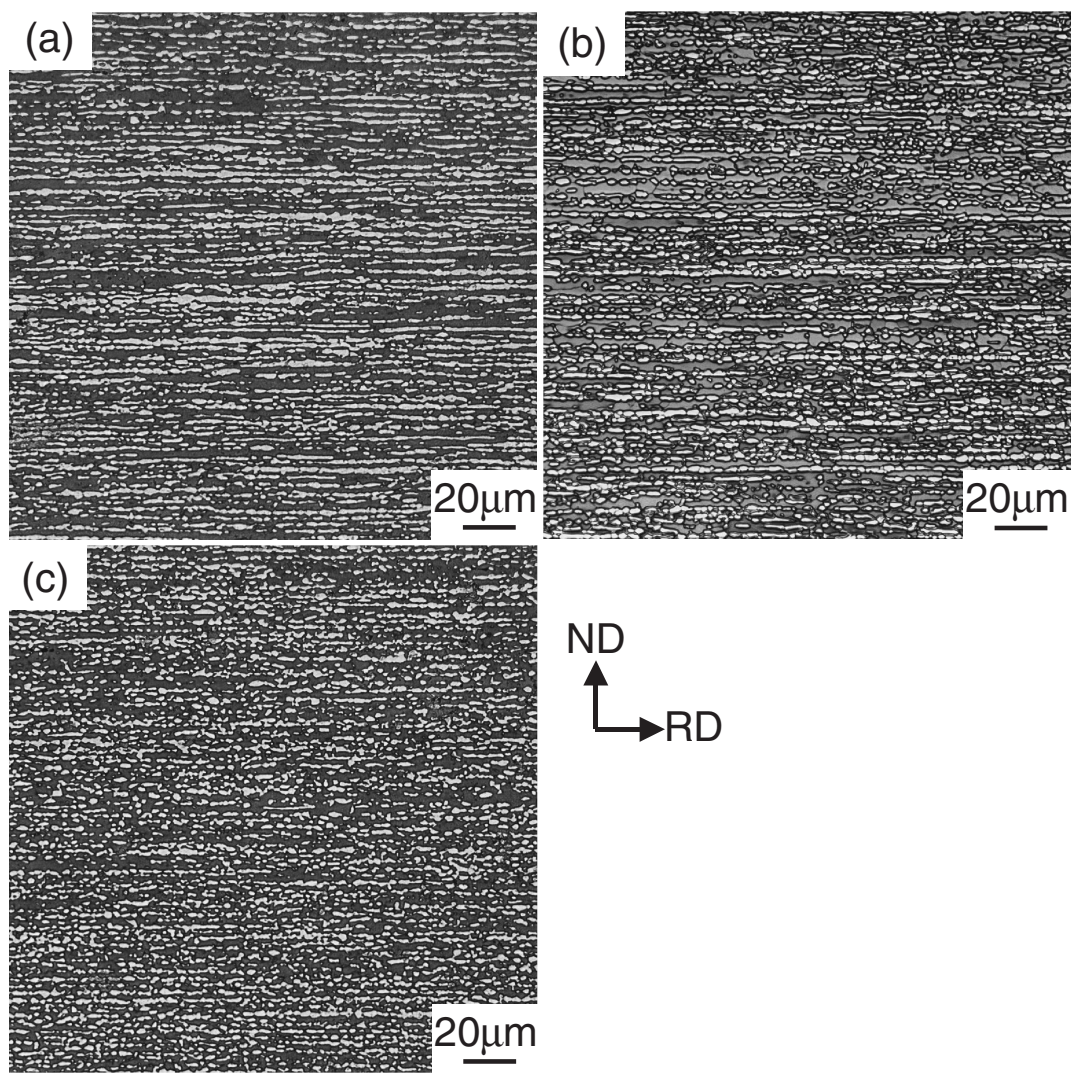

Fig. 5 Optical microstructures on the longitudinal section of final annealed sheets with (a) $67 \%$, (b) $78 \%$, and (c) $89 \%$ cold-rolling reductions.

stainless steel sheets with different textures, but the improvement of the average $r$-value with the increase in cold-rolling reduction is qualitatively similar to that in the case of ferritic stainless steel sheets. However, the magnitude of anisotropy is large in comparison with the ferritic and austenitic steel sheets, ${ }^{27-29)}$ and there is a characteristic that the $r$-values in the vicinity of the diagonal direction are remarkably high for type 329J4L DSS sheet.

\subsection{Microstructures}

Figure 5 shows the microstructures (TD plane) of the final annealed sheets. These sheets consisted of the $\alpha$ matrix (dark) elongated parallel to the rolling direction and the $\gamma$ phase (white) partially fragmented by the $\alpha$ phase. The result of measuring the fraction of the $\alpha$ matrix of final annealed sheet with $67 \%$ cold-rolling reduction by using the image analysis was about $63 \%$. In this observation, there is no significant 


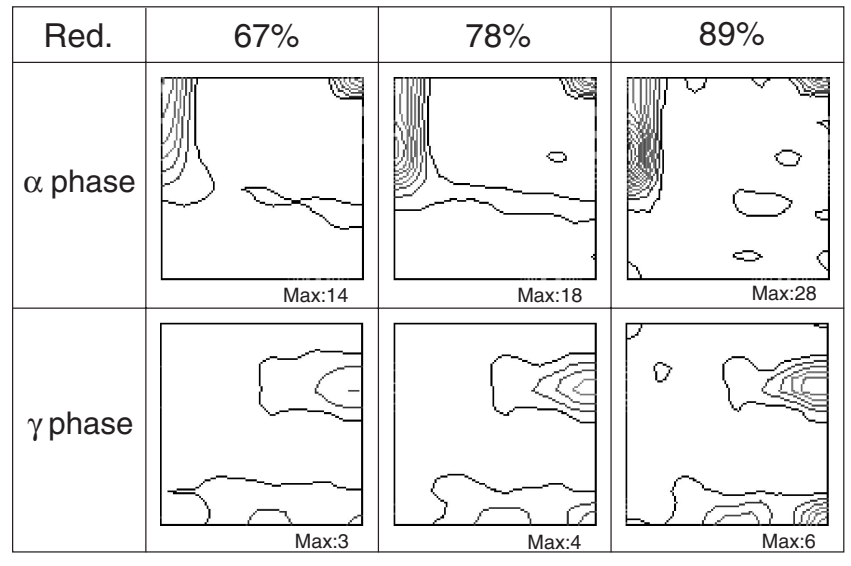

Fig. $6 \varphi_{2}=45^{\circ}$ sections of ODFs in the center layer for final annealed sheets. Contour levels: 2 to 28, step 2 for $\alpha$ phase, and 1 to 6 , step 1 for $\gamma$ phase.

effect of cold-rolling reduction on the microstructures of the final annealed sheets, but the fragmented $\gamma$ phase seems to increase with increasing rolling reduction.

\subsection{Textures}

Figure 6 shows the $\varphi_{2}=45^{\circ}$ sections of the ODFs determined from the ND-section pole figures of the center layers in the final annealed specimens with different coldrolling reductions. The features of a strong $\alpha_{\text {bcc }}$-fiber $(\{100\}\langle 011\rangle-\{211\}\langle 011\rangle-\{111\}\langle 011\rangle)$ and an undeveloped $\gamma$ fiber $(\{111\}\langle 011\rangle-\{111\}\langle 112\rangle)$ in the final annealed sheets are similar to the previous studies. ${ }^{4-10)}$ The orientation density increased with increasing cold-rolling reduction. The main orientation is $\{100\}\langle 011\rangle$ at a $67 \%$ cold-rolling reduction, while it is $\{211\}\langle 011\rangle$ at $78 \%$ and $89 \%$ cold-rolling reductions. From the calculated result of the planar anisotropy of the $r$-value for some ideal orientations, the diagonal direction of the $\{211\}\langle 011\rangle$ component shows a higher $r$ value than the longitudinal and transverse directions. ${ }^{16)}$ It is speculated that the development of $\{211\}\langle 011\rangle$ component in the $\alpha$ phase with an increase in cold-rolling reduction is related to the enhancement of anisotropy by the increased $r$ value in the $45^{\circ}$ direction. The $\gamma$ phase has a $\beta$-fiber texture including major components of $\{112\}\langle 111\rangle$ (Copper-orientation), $\{123\}\langle 634\rangle$ (S-orientation), and $\{011\}\langle 211\rangle$ (Brassorientation), and a $\alpha_{\mathrm{fcc}}$-fiber texture including major components of $\{011\}\langle 100\rangle$ (Goss-orientation) and $\{011\}\langle 211\rangle$. The orientation density in the $\gamma$ phase increases with increasing cold-rolling reduction.

Figure 7 shows the $\varphi_{2}=45^{\circ}$ sections of the ODFs determined from the ND-section pole figures of the surface, quarter, and center layers in the final annealed specimen with a $67 \%$ cold-rolling reduction. Although the $\gamma$ phase exhibits almost no texture change along the thickness direction, the $\alpha$ phase has a significant texture gradient in the thickness direction. Namely, in the $\alpha$ phase, the $\alpha_{\text {bcc }}$-fiber is strongly developed in the quarter and center layers, but in the surface layer the orientation density is weak compared with the other layers, and the Goss orientation $\{011\}\langle 100\rangle$ is slightly formed. Thus, when there is such a texture gradient in the thickness direction, it is impossible to precisely predict the planar anisotropy of the $r$-value from the texture on a

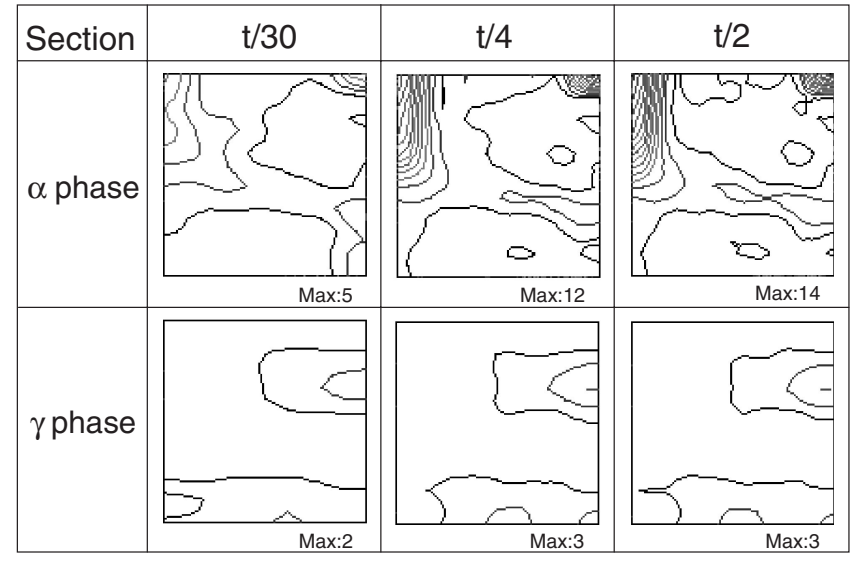

Fig. 7 Partial textures in the surface, quarter, and center layers for final annealed sheet with $67 \%$ cold-rolling reduction. Contour levels: 1 to 14 , step 1 for $\alpha$ and $\gamma$ phases.

particular ND-section. ${ }^{16,20-22)}$ Figure 8 shows the overall ODFs determined from the TD-section pole figures for the $\alpha$ and the $\gamma$ phases in the final annealed sheets with different cold-rolling reductions. In this figure, the overall ODFs are presented in $\varphi_{2}$ section through Euler space in order to show in particular the fiber texture of the $\gamma$ phase clearly. In terms of the type of texture, the overall ODFs are the same as the partial ODFs in various locations through the sheet thickness; the maximum orientation densities of the overall ODFs have a tendency to be weak in comparison with those of the partial ODFs in the center layers. This proves that the texture gradient in the thickness direction is taken into account in the overall texture.

\subsection{Predictions of $\boldsymbol{r}$-value}

Figure 9 shows the planar anisotropies of the $r$-values measured experimentally and calculated using the FC model from an overall texture of the $\alpha$ or $\gamma$ phase. The predicted results obtained by the RC23, RC1323, and RC121323 models are compared with the experimental results in Figs. 10, 11, and 12, respectively. The anisotropies of the calculated results using any model from the overall texture of the $\gamma$ phase are smaller than those obtained by experiments. The calculated values using the FC model for the $\alpha$ phase also significantly differ from the experimental values in all conditions of cold-rolling. The predicted results obtained by the RC23 and RC121323 models for the $\alpha$ phase overestimated the $r$-value in the vicinity of the $0^{\circ}$ and/ or $90^{\circ}$ directions in comparison with the RC1323 model. When the RC1323 model for the $\alpha$ phase, which was the most suitable model for ferritic steel sheets, ${ }^{16,20,21,23,24)}$ is used, the calculated results become much closer to the experimental values in comparison with the other models. The RC1323 model with relaxed shear strains for the $\alpha$ phase gives a better agreement than the FC models assuming homogeneous deformation in all grains for the $\alpha$ and $\gamma$ phases. For the $\{211\}\langle 111\rangle$ and $\{110\}\langle 111\rangle$ glide systems in the $\alpha$ phase, CRSS ratios of $0.95^{23,24)}$ and $1.1^{6,16,20,21)}$ have been reported to be suitable for single-phase ferritic steels. The result indicates that the prediction for the $45^{\circ}$ direction has a tendency toward overestimation when only a $\{110\}$ slip system is used for calculation, so that we should 
(a)

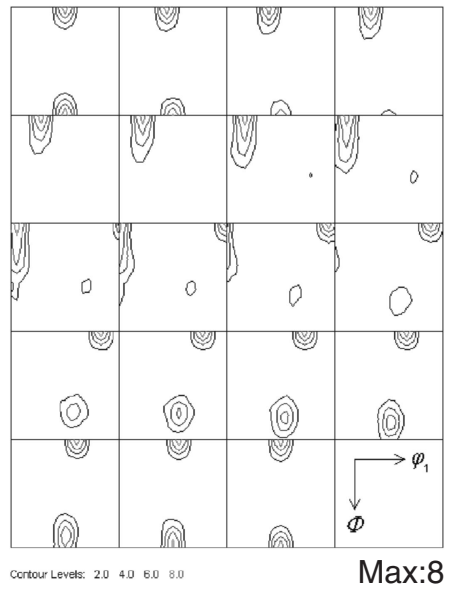

(b)

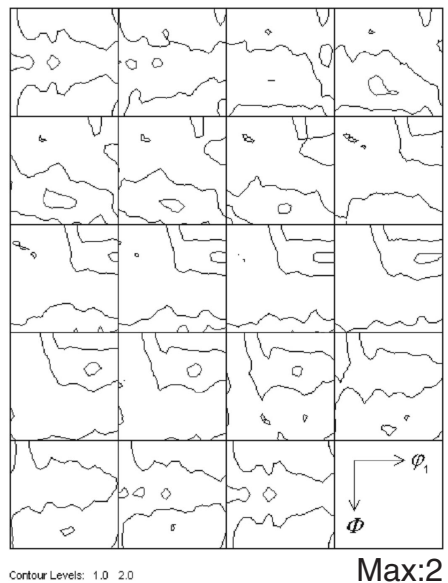

(c)

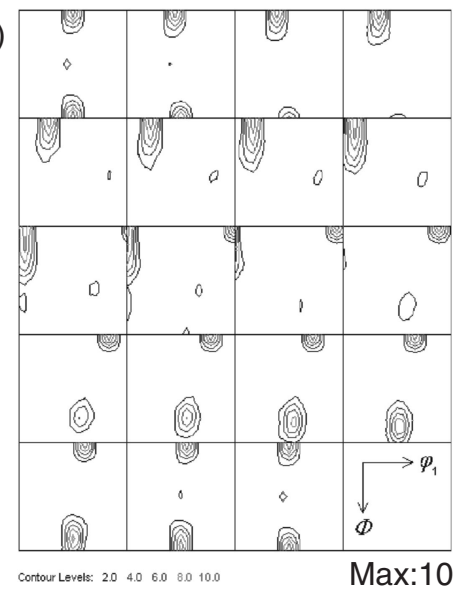

(d)

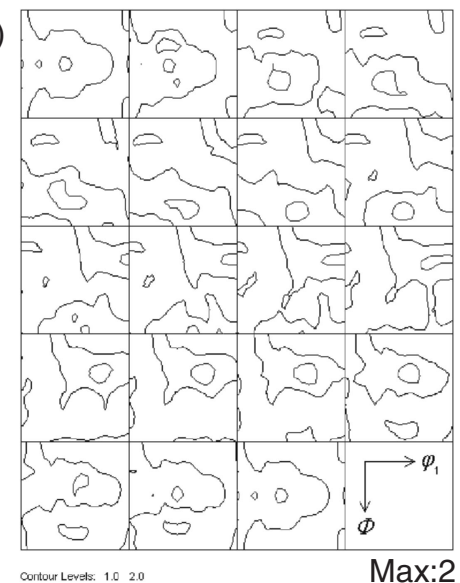

(e)

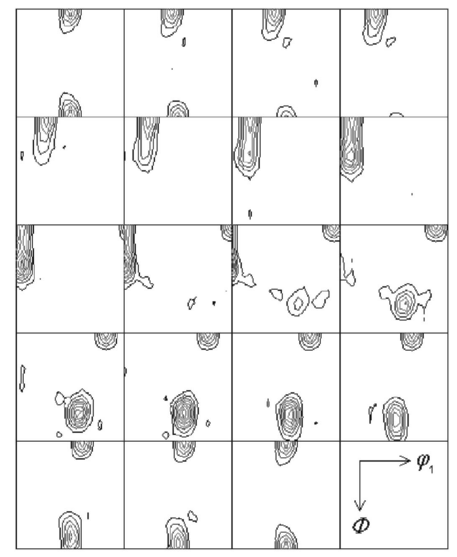

Contour Levels: 20406080100120140180018.0 Max:18

(f)

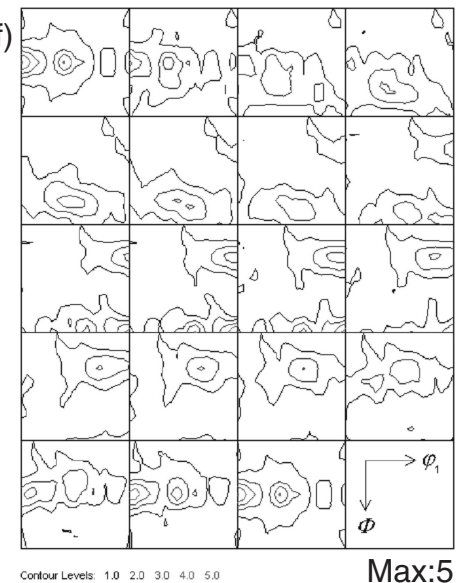

Fig. 8 Overall textures through sheet thickness for (a), (c), and (e) $\alpha$ phase, and (b), (d), and (f) $\gamma$ phase in final annealed sheets with (a) and (b) 67\%, (c) and (d) 78\%, and (e) and (f) 89\% cold-rolling reductions. Contour levels: 2 to 18, step 2 for $\alpha$ phase, and 1 to 5, step 1 for $\gamma$ phase.
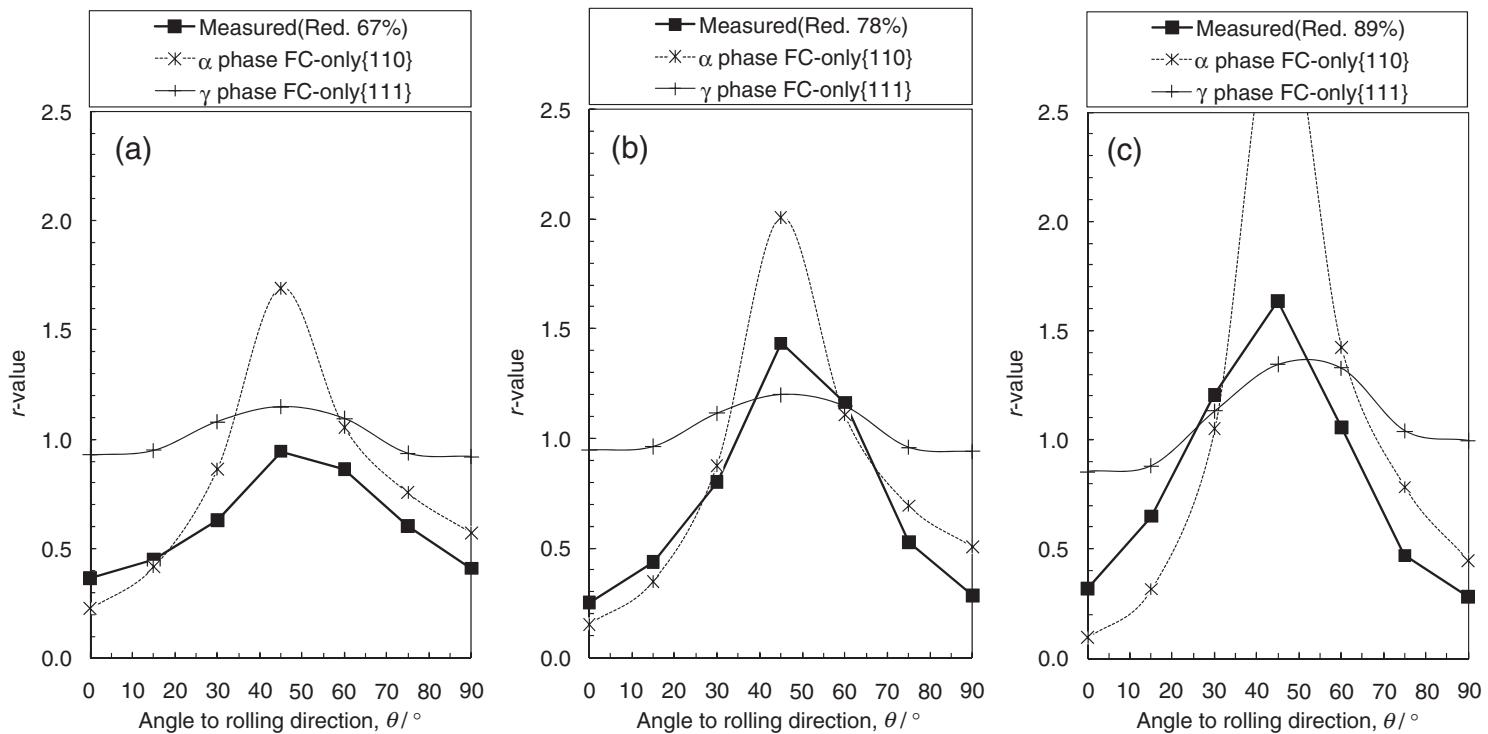

Fig. 9 Planar anisotropy of $r$-value measured and calculated using FC model from the overall texture for final annealed sheets with (a) $67 \%$, (b) $78 \%$, and (c) $89 \%$ cold-rolling reduction.

consider both the $\{110\}$ and $\{211\}$ slip systems for the $\alpha$ phase in type 329J4L DSS sheet like in single-phase ferritic steels. From the calculations by CRSS ratios of $0.9,1.0$, and 1.1 , the most suitable ratio cannot be determined, but a
CRSS ratio of 1.0 or 1.1 provides the calculated value close to the experimental value. The comparisons in $r_{\mathrm{m}}$ and $A_{r}$ between the experimental result and the calculated one by some models are shown in Fig. 13. From these results, it 

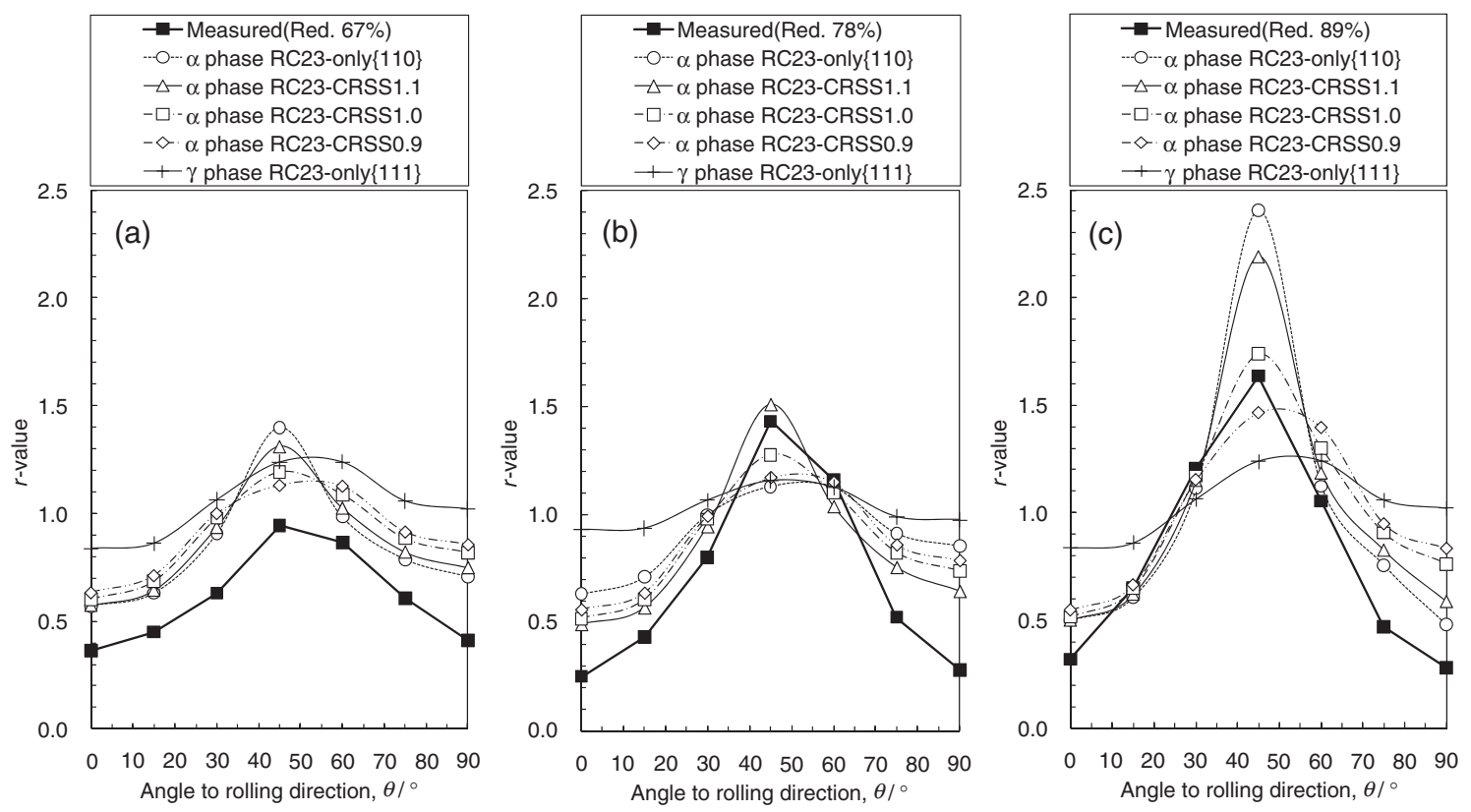

Fig. 10 Planar anisotropy of $r$-value measured and calculated using RC23 model from the overall texture for final annealed sheets with (a) $67 \%$, (b) $78 \%$, and (c) $89 \%$ cold-rolling reduction.
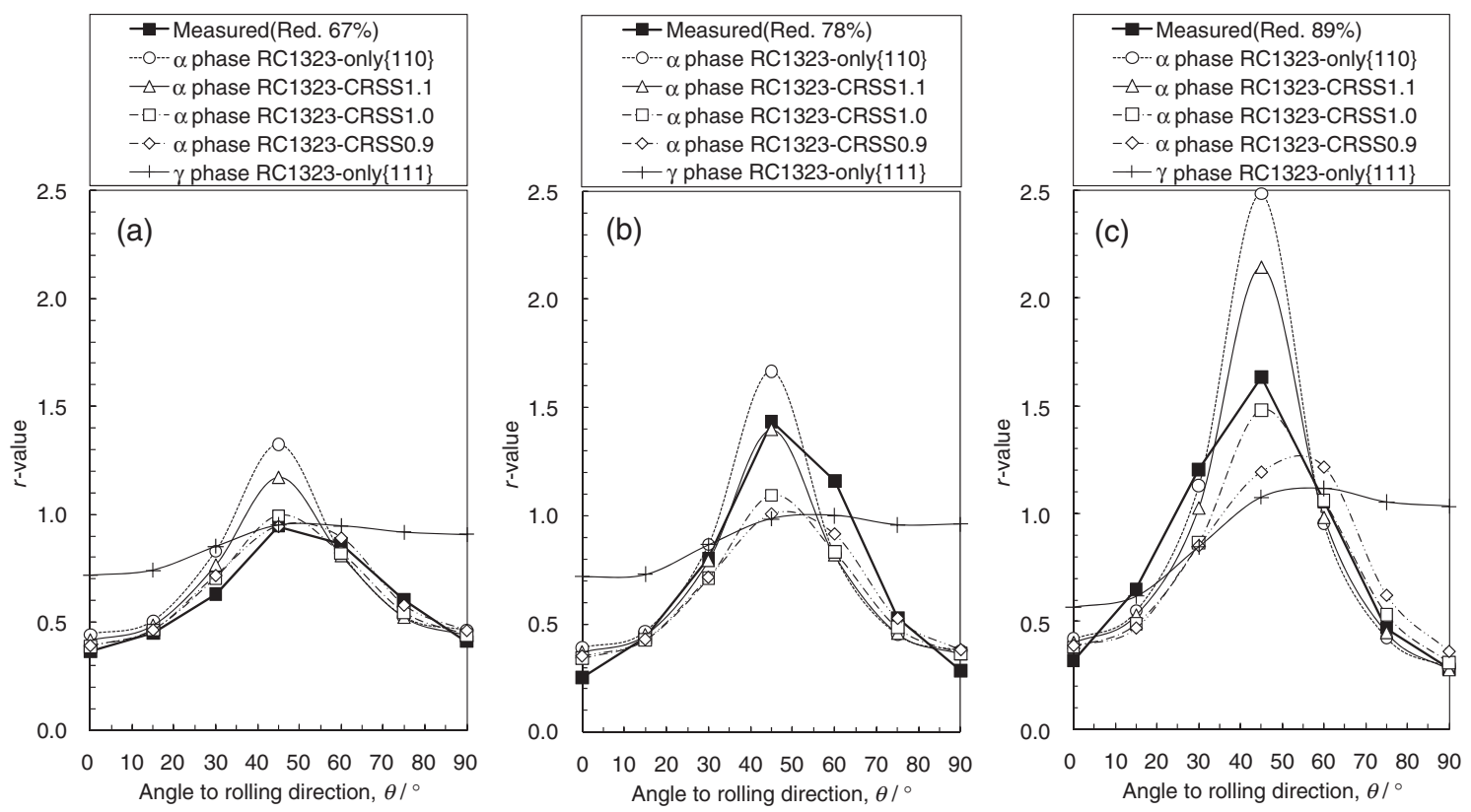

Fig. 11 Planar anisotropy of $r$-value measured and calculated using RC1323 model from the overall texture for final annealed sheets with (a) $67 \%$, (b) $78 \%$, and (c) $89 \%$ cold-rolling reduction.

should be noted that among the models investigated in this study, the RC1323 model with a CRSS ratio of 1.1 for the $\alpha$ phase gives comparatively good coincidence with the measured values. In order to provide the best prediction for the $r$-value, i.e. in order to determine both the model and CRSS ratio, it is necessary to make a comparison with a number of experimental data. However, because the same $\mathrm{RC}$ model for the $\alpha$ matrix is suitable in spite of differences in the existence of the secondary phase and the morphology of grains in type 329J4L DSS sheet and ferritic single phase sheet, it is suggested that slip deformation in the $\alpha$ matrix which is softer than the $\gamma$ phase, and the volume fraction are more dominant in tensile behavior at relatively low strain in type 329J4L DSS sheet, and that inhomogeneous deformation occurs in the $\alpha$ phase similar to single-phase ferritic steels.

Furthermore, much attention should be paid to the direction where the difference between the experimental and calculated values is remarkable, especially to the diagonal direction. The microstructure on the rolling plane in the center layer is shown in Fig. 14. As both the $\alpha$ and $\gamma$ phases are elongated along the longitudinal direction unlike single-phase materials, a shape factor of the $\alpha$ phase or grain boundaries should be considered for DSS sheets. From the viewpoint of the morphological contribution, Moverare et $a l .{ }^{8)}$ presented that for loading at the $45^{\circ}$ direction, the 

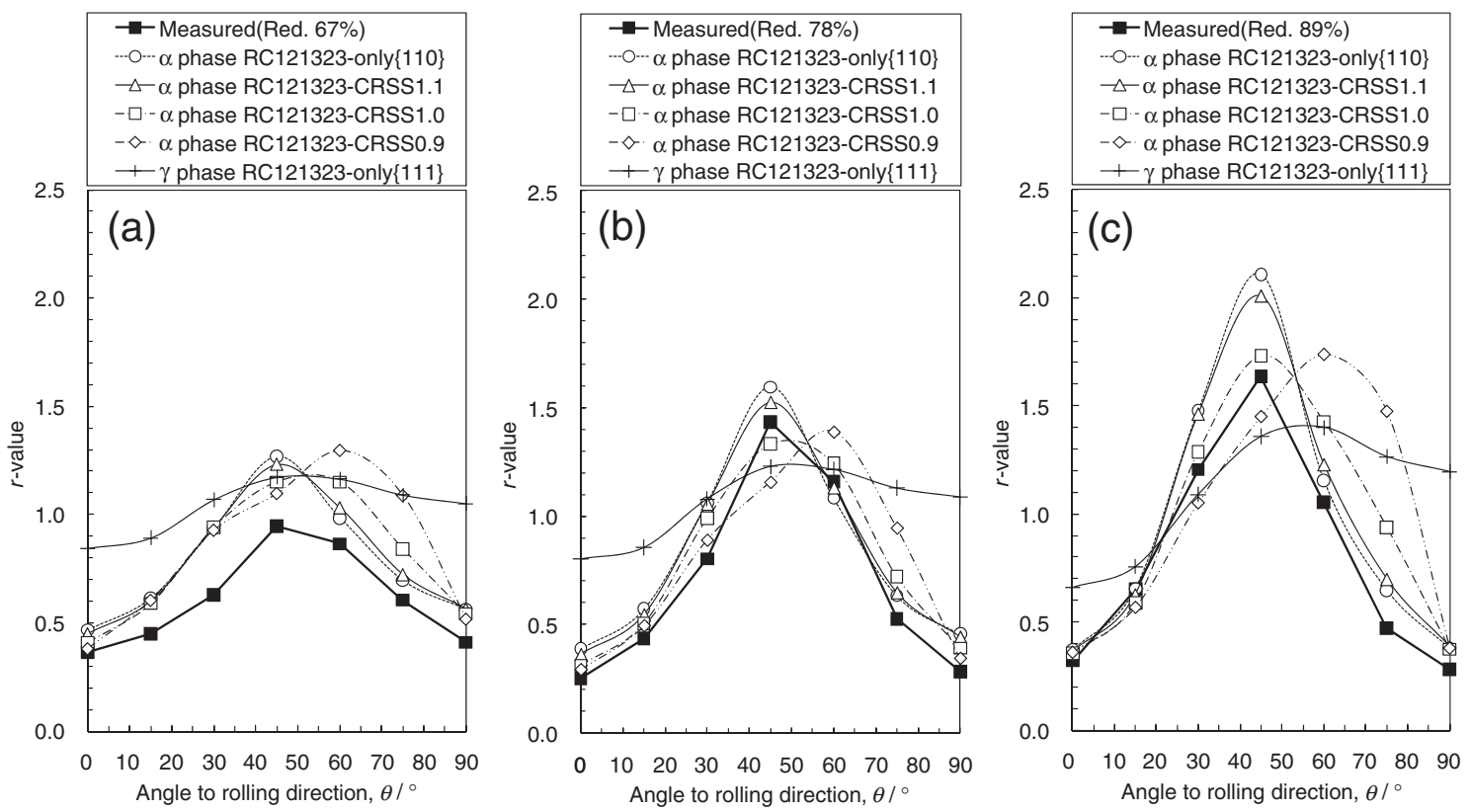

Fig. 12 Planar anisotropy of $r$-value measured and calculated using RC121323 model from the overall texture for final annealed sheets with (a) $67 \%$, (b) $78 \%$, and (c) $89 \%$ cold-rolling reduction.
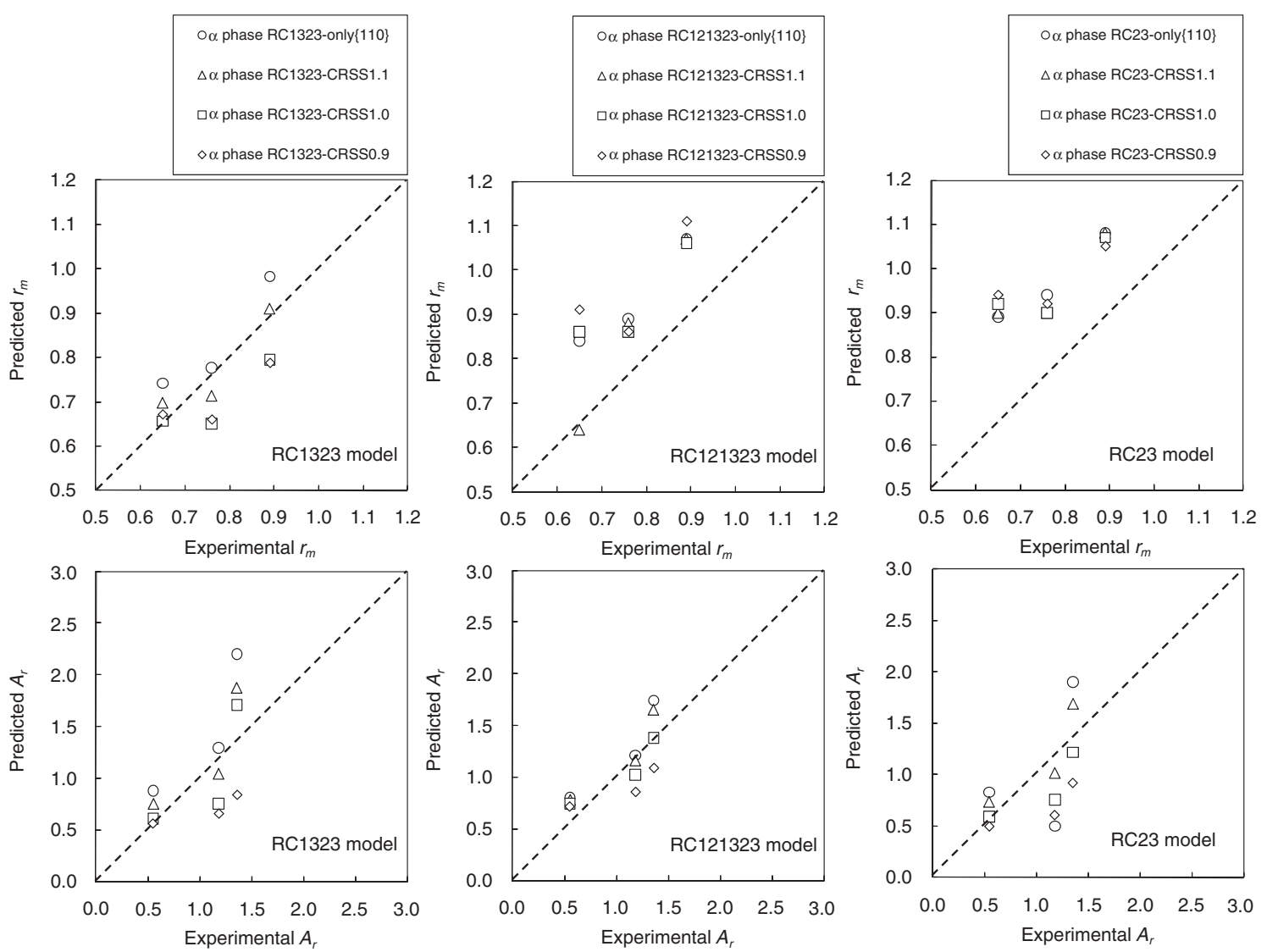

Fig. 13 Correlations between experimental and calculated average $r$-value $\left(r_{\mathrm{m}}\right)$ and anisotropic parameter $\left(A_{r}\right)$.

direction of maximum shear stress was oriented to the same direction as the elongated microstructure and the slip lines extended over a greater distance compared with loading at the rolling and transverse directions. Further investigations in consideration of the morphology and observations of the deformation structure are required in future to realize the precise prediction of plastic anisotropy in DSS sheets.

\section{Conclusions}

In this study, the effect of cold-rolling reduction on texture and planar anisotropy of the $r$-value was investigated in type 329J4L DSS sheet. Moreover, the possibility of predicting the planar anisotropy of the $r$-value on the basis of the Taylor theory was examined in consideration of the texture 


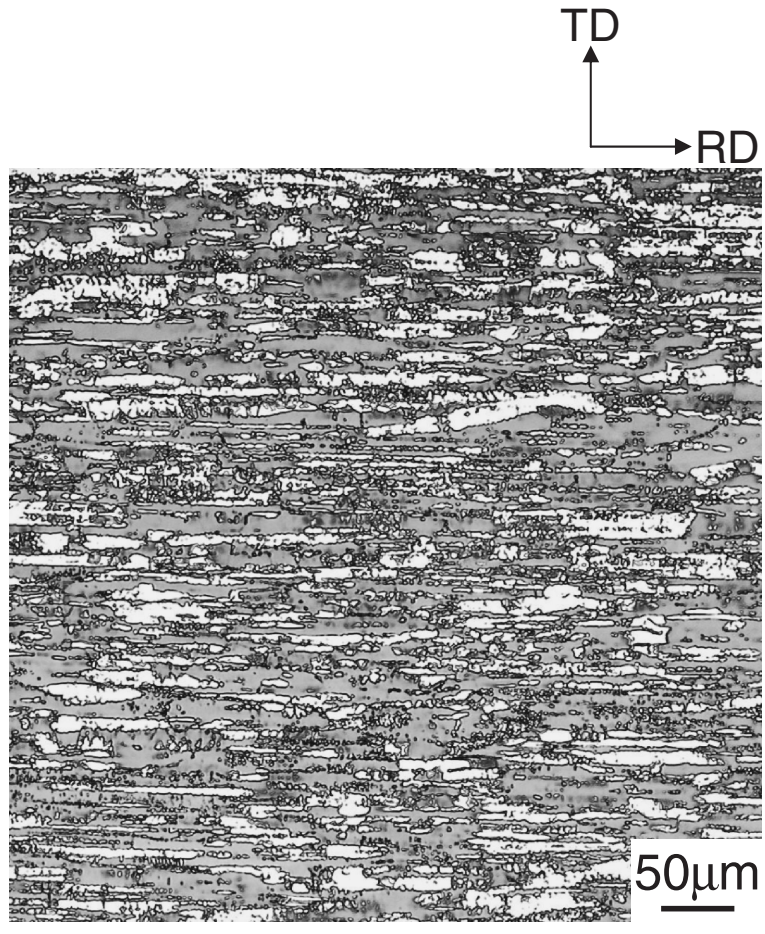

Fig. 14 Optical microstructure on the mid-thickness section of final annealed sheets with $78 \%$ cold-rolling reduction.

gradient in the thickness direction. The main results can be summarized as follows:

(1) With an increase in cold-rolling reduction, the average $r$-value $r_{\mathrm{m}}$ increased, and a strong directionality appeared, having the maximum value in the diagonal direction.

(2) The $\alpha$ phase had an extremely strong $\alpha_{\mathrm{bcc}}$-fiber texture, and the $\gamma$ phase had $\beta$ - and $\alpha_{\mathrm{fcc}}$-fiber textures. With an increase in rolling reduction, the orientation density became higher in both phases. The texture gradient in the thickness direction was more remarkable in the $\alpha$ phase than in the $\gamma$ phase.

(3) The $r$-values calculated from the overall texture through sheet thickness of the $\alpha$ phase using the Taylor theory with the RC1323 model and a CRSS ratio of 1.1 were in quite good agreement with the experimental $r$-values like single-phase ferritic steels, and it was concluded that the $\alpha$ matrix was dominant in tensile deformation of type 329J4L DSS sheet.

\section{Acknowledgment}

One of the authors (J.H) would like to thank Mr. Kazuyuki Agata and Mr. Noriaki Yagi, Nippon Steel \& Sumikin Stainless Steel Corp., for the X-ray measurements.

\section{REFERENCES}

1) J. Charles: Steel Re. 79 (2008) 455-465.

2) J.-O. Nilsson: Mater. Sci. Technol. 8 (1992) 685-700.

3) N. Ono, J. Hamada, I. Muto and T. Komori: CAMP-ISIJ 16 (2003) 550.

4) G. Fargas, N. Akdut, M. Anglada and A. Mateo: ISIJ Int. 48 (2008) 1596-1602.

5) J. Hamada and N. Ono: Mater. Trans. submitted.

6) H. Inoue, J. Hamada and T. Goto: Yield strength and microstructure in steels-Results of experiment and simulation-, (ISIJ, Tokyo, 2007) pp. 44-49.

7) W. B. Hutchinson, K. Ushioda and G. Runnsjö: Mater. Sci. Technol. 1 (1985) 728-731

8) J. J. Moverare and M. Odén: Metall. Mater. Trans. A 33A (2002) 57-71.

9) J. Keichel, J. Foct and G. Gottstein: ISIJ Int. 43 (2003) 1781-1787.

10) J. Keichel, J. Foct and G. Gottstein: ISIJ Int. 43 (2003) 1788-1794.

11) T. Maki, T. Furuhara and K. Tsuzaki: ISIJ Int. 41 (2001) 571-579.

12) T. Maki: Netsu Shori 39 (1999) 5-11.

13) X. Huang, K. Tsuzaki and T. Maki: Scr. Metall. Mater. 33 (1995) 341346.

14) H. Inoue and N. Inakazu: J. Japan Inst. Metals 58 (1994) 892-898.

15) H. Inoue and T. Takasugi: Z. Metallkd. 92 (2001) 82-88.

16) J. Hamada, K. Agata and H. Inoue: Mater. Trans. 50 (2009) 752-758.

17) G. I. Taylor: J. Inst. Metals 62 (1938) 307-324.

18) H. Honneff and H. Mecking: Proc. 5th Int. Conf. on Textures of Materials, ed. by G. Gottstein and K. Lücke, (Springer-Verlag, Berlin, 1978, Vol. 1) pp. 265-275.

19) H. Inoue and N. Inakazu: J. Japan Inst. Light Met. 44 (1994) 97-103.

20) H. Inoue: Recrystallization Textures and Their Application to Structure Control, (ISIJ, Tokyo, 1999) pp. 174-181.

21) H. Inoue, K. Sekine and T. Hasegawa: Proc. 76th JSME Fall Annual Meeting, (The Japan Society of Mechanical Engineers, 1998) pp. 4748.

22) H. Inoue and T. Takasugi: Mater. Trans. 48 (2007) 2014-2022.

23) D. Daniel and J. J. Jonas: Metall. Trans. A 21A (1990) 331-343.

24) R. Schouwenaars, P. Van Houtte, E. Aernoudt, C. Standaert and J. Dilewijns: ISIJ Int. 34 (1994) 366-372.

25) H. J. Bunge: Kristall und Technik 5 (1970) 145-175.

26) H. J. Bunge: Texture Analysis in Materials Science, (Butterworths, London, 1982).

27) T. Sawatani, K. Shimizu, T. Nakayama and M. Miyoshi: Tetsu-toHagané 63 (1977) 843-854.

28) R. M. Davison: Metall. Trans. 5 (1974) 2287-2294.

29) T. Sato, Y. Tashiro, Y. Honda, M. Nishida and A. Chiba: ISIJ Int. 38 (1998) 640-646. 